\title{
Filling Behavior of Polymer Melt in Micro Injection Molding for V-Grooves Pattem
}

\author{
Moo Sun Kim ${ }^{\mathrm{a}}$, Seung Mo Kim ${ }^{\mathrm{b} *}$ \\ V-Groove 패턴을 위한 마이크로 사출성형의 폴리머 멜트 충전 거동 \\ 김무선 ${ }^{\mathrm{a}}$, 김승모 ${ }^{\mathrm{b} *}$ \\ ${ }^{a}$ Future Metropolitan Railroad System Research Division, Metropolitan transportation Research Center, Korea Railroad Research \\ Institute, 176 Cheoldo bakmulkwan-ro, Uiwang city, Gyeonggi province, Republic of Korea \\ ${ }^{b}$ School of Mechanical Engineering, Korea University of Technology and Education, 1600 Chungjeol-ro, Byeongchun-myun, Cheonan \\ city, Chungnam province, Republic of Korea
}

\begin{tabular}{|c|c|c|c|}
\hline \multicolumn{4}{|c|}{ ARTICLE INFO } \\
\hline \multicolumn{4}{|c|}{ Article history: } \\
\hline Received & 22 & March & 2014 \\
\hline Revised & 30 & April & 2014 \\
\hline Accepted & 7 & May & 2014 \\
\hline
\end{tabular}

Keywords:

Injection molding

Polymer melt

Micro pattern

Filling behavior

V-groove

\begin{abstract}
This study uses two numerical approaches to analyze the filling behavior of micro patterns on micro-injection molding for $\mathrm{V}$-grooves pattern which cannot be simulated with conventional CAE packages. The parametric studies have been performed to examine the fidelity of micro patterns with respect to temperature, pressure, inlet velocity and pattern location on the mold according to the boundary condition from the macro pressure and velocity data which can be obtained by conventional CAE packages. Through these numerical approaches, the filling behavior of polymer melt in micro patterns can be understood, the quality of replication can be predicted, and the $\mathrm{V}$-groove pattern can be shaped uniformly during the process of injection molding.
\end{abstract}

\section{Introduction}

The polymer products have many potential applications in modern component industry where IT, BT and NT rise up as a new leading technology. Especially, the new approach of injection molding is proposed to manufacture the micro features on the large surface, instead of conventional micro patterning method such as photo-lithography ${ }^{[1,2]}$. Through the Injection molding process, the micro polymer patterning process with high efficiency and low cost can be realized. However, the conventional CAE packages such as C-Mold and MoldFlow are based on a generalized hele-shaw (GHS) model in which xy-dimensional elements are used to represent the three-dimensional geometry neglecting the inertia and the gap-wise velocity component for polymer melt flow in the thin plate ${ }^{[3,4]}$. Due to these limits of GHS model, the filling behavior of micro pattern can not be predicted. Therefore, a set of the governing equations which is composed of the conservation of mass, momentum, energy and corresponding constitutive equations should be solved simultaneously to consider such out-of-plane phenomena when molding with micro features on the large surface. Injection molding for plastic processing consists of three distinctive stages, such as filling, packing and cooling stages. When molding a thin-wall

* Corresponding author. Tel.: +82-41-560-1159

Fax: $+82-41-560-1253$

E-mail address: smkim@koreatech.ac.kr (Seung Mo Kim). 
cavity, the effect of packing by holding pressure on the replication is less and the micro patterning is generally completed during the filling stage. But, the filling behavior into V-grooves is yet to be clarified, and also the micro injection molding on large surface is difficult to predict in conventional analysis. Hence, it is desirable to confirm the analysis using a new simulation approach. In this study, a local analysis based on Navier-Stokes equation is performed according to the specific local boundary condition from the global analysis to thoroughly understand the filling flow in micro patterns. An understanding of the process

involving the filling of polymer melt is important not only for understanding but also for further improving.

\section{Simulation Procedure}

\subsection{Goveming Equation}

In this study, the polymer melt flow is considered to be two-dimensional because continuous V-groove patterns are considered for micro scale patterns and the width of the mold is assumed to be much larger than the thickness. It was also assumed that the flow is incompressible ${ }^{[5,6]}$. For the polymer melt flow, the governing equations consist of mass, momentum (Navier-Stokes equation) and energy conservation equations ${ }^{[7]}$. Due to the injection molding is processing in non-isothermal condition between mold surface and polymer melts, the energy conservation equation is also considered.

Mass, momentum, and energy conservation can be written as follows:

$$
\begin{aligned}
& \frac{\partial \rho}{\partial t}+\rho \frac{\partial u_{i}}{\partial x_{i}}=0 \\
& \rho \frac{\partial u_{i}}{\partial t}+\rho u_{j} \frac{\partial u_{i}}{\partial x_{j}}=\frac{\partial}{\partial x_{j}} \sigma_{j i}(u)+\rho f_{i} \\
& \rho C_{p}\left(\frac{\partial T}{\partial t}+u_{i} T_{, i}\right)=\left(K T_{, i}\right)_{, i}+Q+\Phi
\end{aligned}
$$

where $\sigma_{i j}=-p \delta_{i j}+2 \eta d_{i j}$ and $d_{i j}=\frac{1}{2}\left(\frac{\partial u_{i}}{\partial x_{j}}+\frac{\partial u_{j}}{\partial x_{i}}\right) \quad(\mathrm{i}=1,2$ and $\mathrm{j}=1,2$ ). Here, $t$ is the time, $u_{i}$ is the velocity component in $x_{i}$ direction, $\rho$ is the density $\left(1.06 \mathrm{e}^{3} \mathrm{~kg} / \mathrm{m}^{3}\right), \eta$ is the viscosity, $f_{i}$ is the body force component in $x_{i} . \sigma_{i j}$ and $d_{i j}$ denote the stress and the strain tensors. And $T, C_{p}, k, Q$ and $\Phi$ are temperature, specific heat, thermal conductivity, rate of internal heat generation and the viscous dissipation in the fluid, respectively. The viscous dissipation term is the following:

$$
\Phi=2 \eta d_{i j} d_{i j}
$$

Above set of governing equations should be solved numerically. Finite element method was used to discretize the equations. The rectangular 4-node element based on penalty formulation was employed ${ }^{[8]}$. In order to deal with the moving boundary problem, the volume of fluid (VOF) method was used to trace the free surface. In the VOF method, the fractional volume is defined for element variable. The fractional volume is used to divide the total domain into the fluid occupied and empty regions. The values of the fractional volumes in fully filled cells, partially filled cells, and empty cells are given by unity, between zero and unity, and zero, respectively. The fractional volume is then computed and updated at each time step using the advection equation as follows

$$
\frac{\partial C}{\partial t}+u_{i} \frac{\partial C}{\partial x_{i}}=0
$$

where $C$ is the color function (fractional volume) ${ }^{[9]}$. The process of free surface construction consists of three steps: first, the flow field is solved at one time step. Then the fluid volume flux from one element to the neighboring element is calculated ${ }^{[10]}$. Finally, the fractional volume is updated. This process for each time step is repeated until the desired time is reached.

\subsection{Rheological behavior}

The Cross-WLF model for viscous flow is adopted to describe the non-Newtonian behavior of polymer melt. For the given temperature $T$, the corresponding viscosity function $\eta\left(I_{2}, T\right)$ takes the following form ${ }^{[11,12]}$ :

$$
\eta\left(I_{2,} T\right)=\frac{\eta_{0}(T)}{1+\left(\eta_{0}(T) \cdot I_{2} / \tau\right)^{1-n}}
$$


Table 1 Data of constants in the Cross-WLF model for PMMA

\begin{tabular}{c|c|c|c|c|c}
\hline \hline $\mathrm{n}$ & $\tau(\mathrm{Pa})$ & $\mathrm{A}_{1}(\mathrm{~K})$ & $\mathrm{A}_{2}(\mathrm{~K})$ & $\mathrm{D}_{1}(\mathrm{~Pa} \cdot \mathrm{s})$ & $\mathrm{D}_{2}(\mathrm{~K})$ \\
\hline 0.3973 & 35607 & 31.081 & 51.6 & $6.13 \mathrm{e}+12$ & 377.15 \\
\hline
\end{tabular}

where $\eta_{0}=D_{1} \exp \left[-A_{1}\left(T-D_{2}\right) /\left(A_{2}+T-D_{2}\right)\right]$ for $T \geq D_{2}$ and $T<D_{2}$ for $\eta_{0}=\infty$. Here, $D_{1}, D_{2}, A_{1}, A_{2}$ and $\tau$ are constant values that should be determined from experimental measurements for a specific polymer. The data of constants used in simulation for PMMA (polymethylmethacrylate) are in Table 1 .

\subsection{Micro-scale effect}

Even though the viscosity of injection molding is known as relatively high value from the Cross-WLF viscosity model, the surface tension effect and contact angle boundary are considered due to the large surface-to-volume ratio on micro-scale configurations ${ }^{[13]}$. The surface tension effect was considered by Brackbill's CSF model formulation ${ }^{[14]}$ which converts surface force to body force as

$$
F=\frac{\sigma}{[C]} \kappa \nabla C, \kappa=-(\nabla \cdot n), n=\frac{\nabla C}{|\nabla C|}
$$

where $[C]$ denotes the jump of $C$ across the interface. Here, $\sigma$ is the surface tension coefficient $(29.7 \mathrm{mN} / \mathrm{m})$ and $\kappa$ is the curvature of the interface. The static contact angle which is assumed by $70^{\circ}$ for wall adhesion condition based on the empirical data is applied in numerical analysis.

\subsection{Numerical Approach}

Micro and macro flows are coexist in micro injection molding ${ }^{[15,16]}$. The micro flow in $\mathrm{V}$-groove pattern on mold wall and the macro flow between top and bottom, its should be analyzed simultaneously to understand the process behavior. But, due to the limited computational memory and time, it is not efficient to simulate the whole domain as micro pattern scale. Therefore, in order to analyze the injection molding with micro features on the large surfaces, a sequential analysis is needed. It includes the global analysis based on GHS model using conventional/commercial CAE packages which dose not consider micro pattern as a computational domain and the

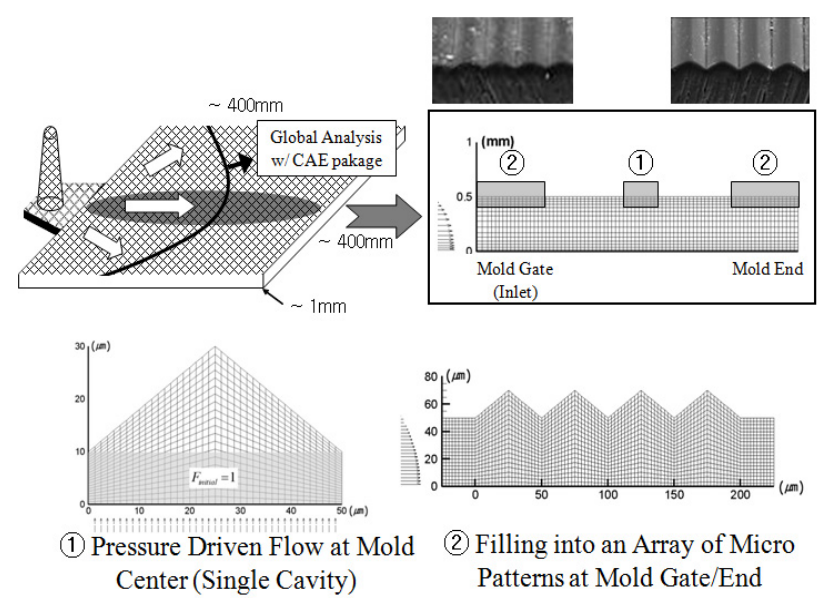

Fig. 1 Schematic diagram of numerical approaches

local analysis based on Navier-Stokes equation for polymer melt flow in micro $\mathrm{V}$-groove pattern on mold wall. While the global polymer flow affects the boundary condition of the local polymer flow, the reverse effect can be negligible due to the fountain flow characteristic of polymer melt. Accordingly these can be conducted step by step from global analysis to local analysis.

As a global analysis, a well-known commercial package can be used to obtain the boundary condition for a local analysis. And then, assuming the local boundary condition from global analysis data such as pressure and velocity profile, the micro scale domain can be analyzed to observe the filling behavior or predict the fidelity of patterns (Fig. 1). From the global analysis data such as pressure and velocity, the local analysis of micro pattern can be simulated by two kinds of approach. The first approach is the pressure driven filling in single cavity according to the macro pressure. And the second approach is the inlet velocity boundary condition in several micro cavities according to the macro velocity profile. In fact, the filling of micro pattern might be affected by the combination of specific portions of two approaches above because the boundary condition cannot be divided perfectly as a pressure and velocity profile. But, the main boundary condition can be characterized depending on the position of mold domain. In this study, the filling behavior of polymer melt in micro pattern was simulated to optimize process parameters using two numerical approaches. 


\section{Results and Discussions}

\subsection{Pressure Driven Filling - Single Cavity}

The pressure driven filling in single cavity assuming the previously filled macro region and the constant pressure boundary condition represents the cases of fast macro inlet velocity, narrower cavity width and single cavity geometry. The boundary condition of pressure driven filling is explained in Fig. 2. The bottom area is initially filled and the flow of micro filling is occurred by the constant pressure boundary conditions. To compare the filling degree of micro cavity, the corresponding fidelity variable is defined as follows.

$$
\text { Degree of Filling }=\frac{(\text { Filled Area })}{(\text { Cavity Area })}
$$

The parametric study is performed with three pressure variation ranging from $100 \mathrm{KPa}$ to $500 \mathrm{KPa}$ at different mold wall temperature $\left(T_{w}\right)$ and initial melt temperature $\left(T_{i}\right)$. Basically, the better filling results are observed in the high pressure and temperature case. Furthermore, the cavity filling
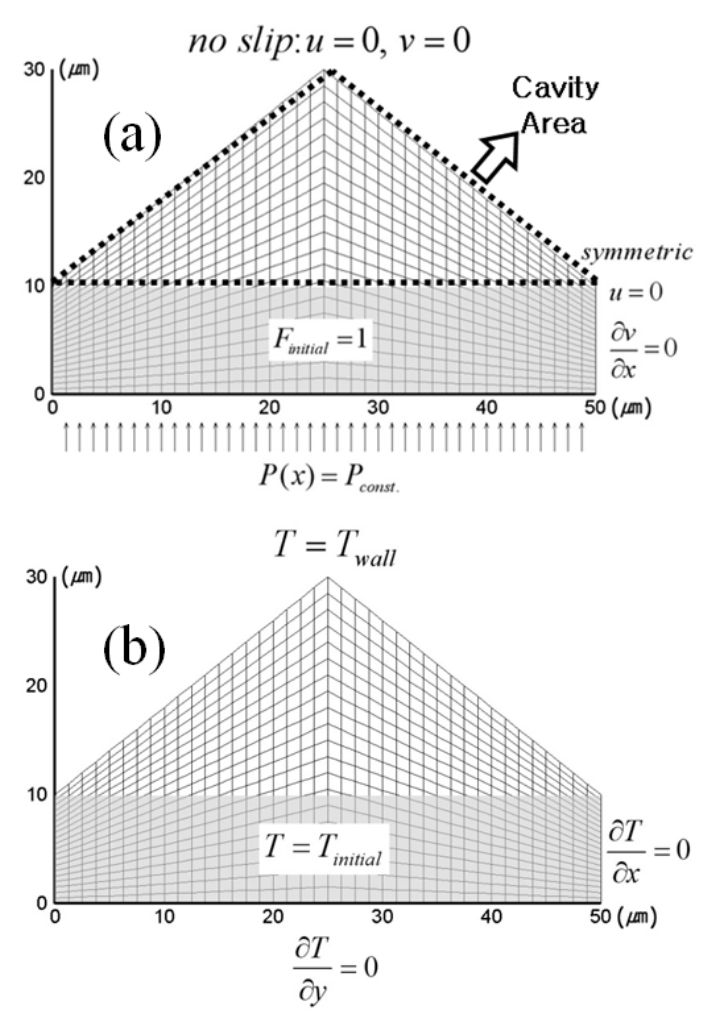

Fig. 2 Pressure driven filling - boundary condition : (a) flow region $\mathrm{BC}$, (b) temperature region $\mathrm{BC}$ is more successful in high initial temperature $\left(T_{w}=150^{\circ} \mathrm{C}\right.$, $\left.T_{i}=200^{\circ} \mathrm{C}\right)$ rather than in high mold temperature $\left(T_{w}=100^{\circ} \mathrm{C}\right.$, $T_{i}=250^{\circ} \mathrm{C}$ ) as shown in Fig. 3, because the temperature distribution of micro cavity is more affected by initial

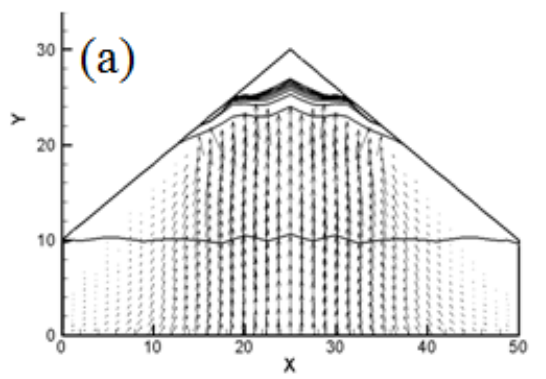

(b) $T_{W}=100 \mathrm{~T} \quad T_{i}=200 \mathrm{~T}$

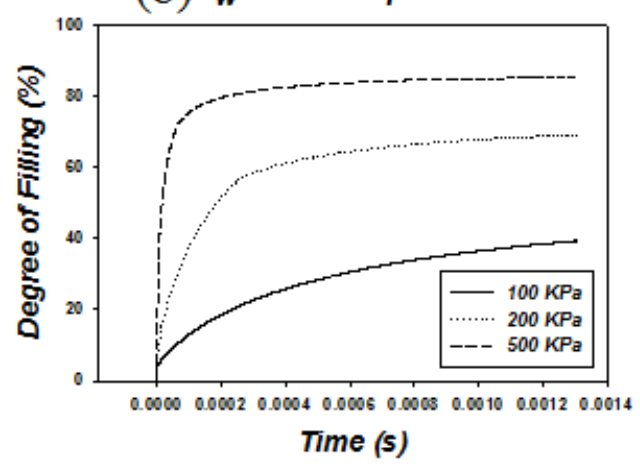

(c) $T_{W}=150 \mathrm{~T} \quad T_{i}=200 \mathrm{~T}$

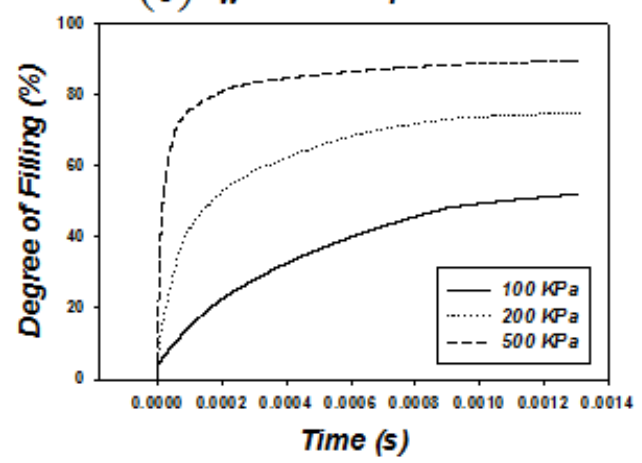

(d) $T_{W}=100 \mathrm{C} \quad T_{i}=250 \mathrm{C}$

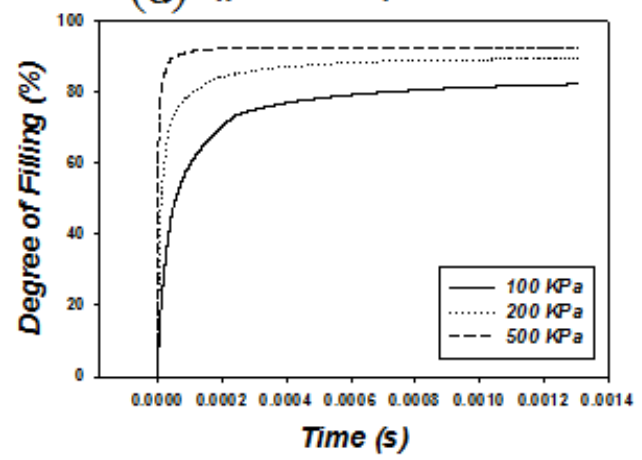

Fig. 3 Degree of filling in single cavity : (a) typical of flow in single cavity shape and (b)-(d) parametric study 
temperature of macro region rather than mold wall temperature. Based on this approach, the filling of single cavity according to specific pressure on the mold can be understood and predicted.

\subsection{Inlet Velocity \& Pattem Location Effect}

The inlet velocity boundary condition instead of pressure
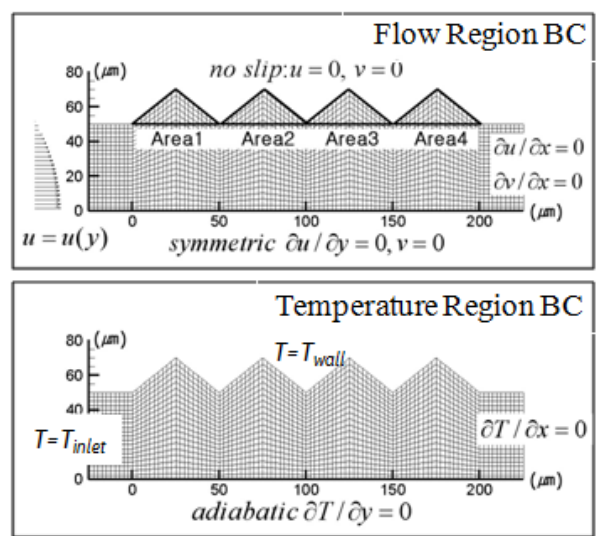

(a)
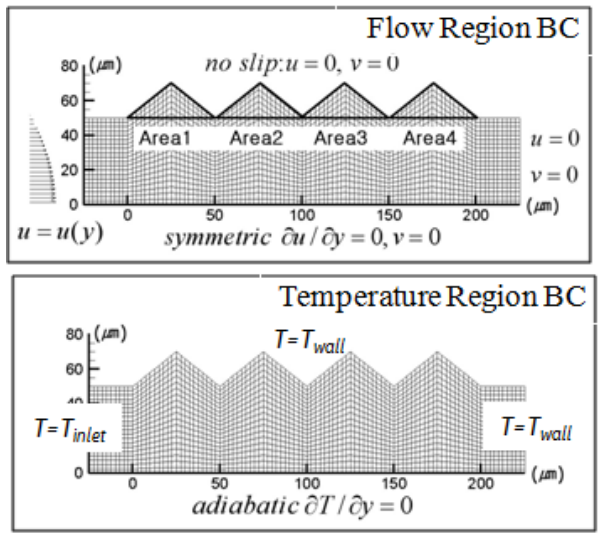

(b)

Fig. 4 Inlet velocity \& pattem location - boundary condition : (a) mold gate, (b) mold end

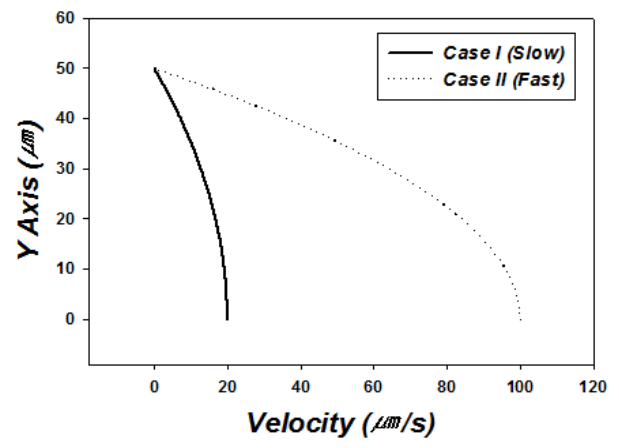

Fig. 5 Inlet velocity profile (fully developed state) driven filling is more suitable in the case of slow macro inlet velocity, wide cavity and continuous several cavities. The whole boundary condition assuming continuous four cavities is explained in Fig. 4. Two kinds of boundary condition such as mold gate and mold end are considered to view the filling effect respect to the pattern location. And two inlet fully developed velocity profile curves which are assumed from the specific macro velocity are shown in Fig. 5.

In this approach, the four micro cavities $(200 \mu \mathrm{m})$ are analyzed as a computational domain samples. And the bottom's boundary is set to be adiabatic condition and the $50 \mu \mathrm{m}$ thickness of macro region which is 2.5 times of cavity's height is set as a computational geometry in order to consider the temperature cooling effect of molds wall.

The parametric study is performed at constant mold wall temperature $\left(T_{w}=100^{\circ} \mathrm{C}\right)$ and initial melt temperature
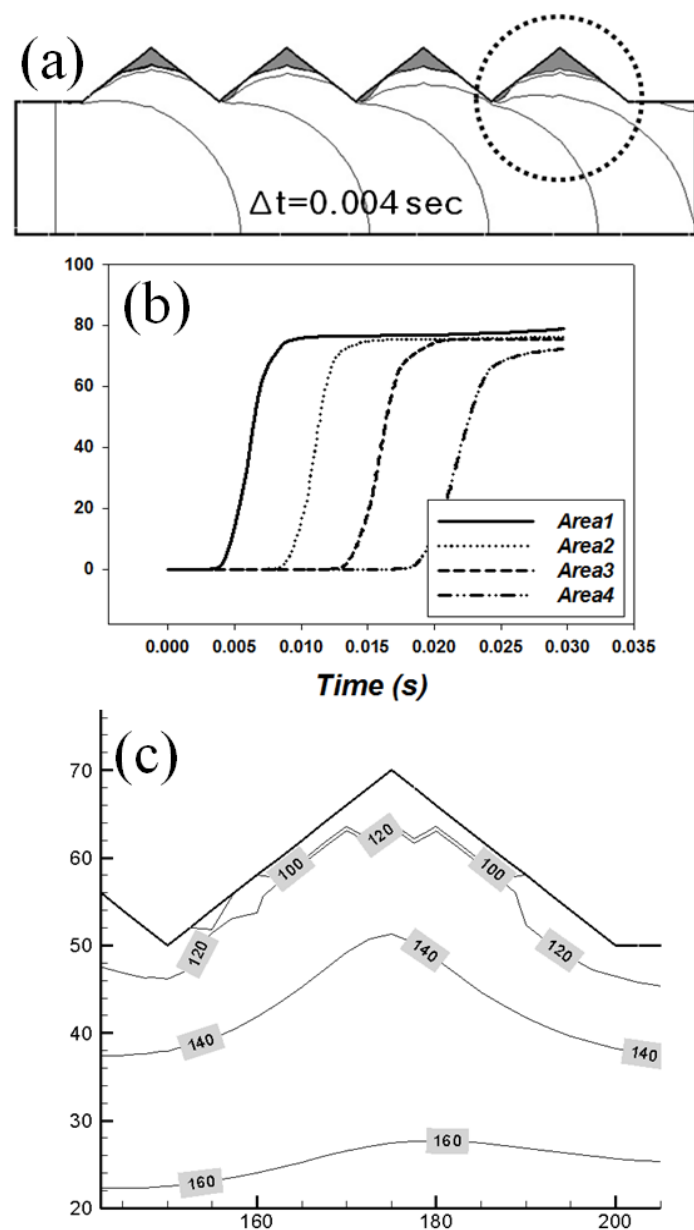

Fig. 6 Inlet velocity (case I) \& mold gate : (a) advancing flow front, (b) degree of filling (average $\sim 78.7 \%$ ), (c) typical temperature contour in circular dotted line region 

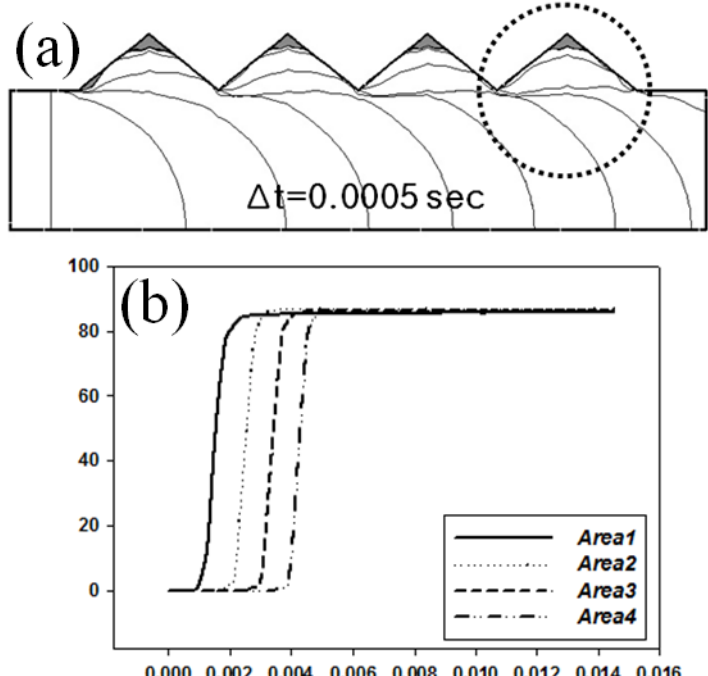

Time (s)

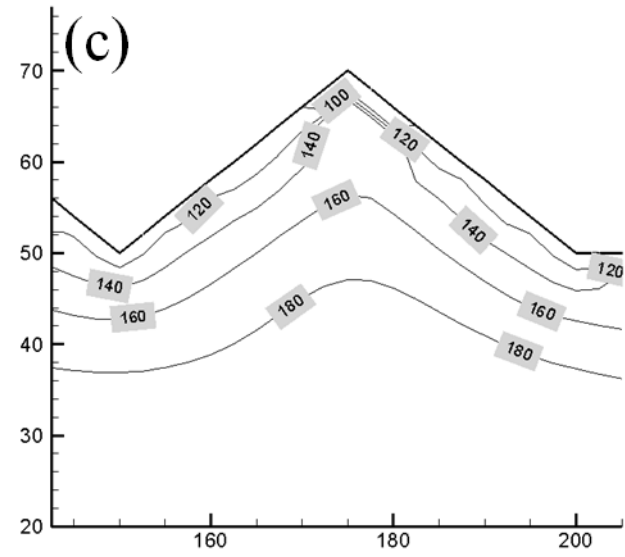

Fig. 7 Inlet velocity (case II) \& mold gate : (a) advancing flow front, (b) degree of filling (average $\sim 84.9 \%$ ), (c) typical temperature contour in circular dotted line region

$\left(T_{i}=200^{\circ} \mathrm{C}\right)$. The degrees of filling for each cavity area is plotted in Figs. 6-9 including the flow front shape respectively with typical time step and the temperature contour of fourth region at the end of cavity filling. Also, the average values of degree of filling for cavities are indicated as a representative value of this numerical approach.

In the case of slow inlet velocity, while the first cavity near the inlet boundary shows better filling due to the absence of backpressure effect in mold gate case (Average $78.7 \%$ ), the fourth cavity shows better filling due to the backpressure from the wall of end case mold (Average $\sim 84.9 \%$ ). But, as the inlet speed is faster, the degree of filling of three cavities become more uniform and the backpressure effect becomes more dominant in fourth cavity due to the low viscosity at a few temperature drop during filling process. As the result, almost
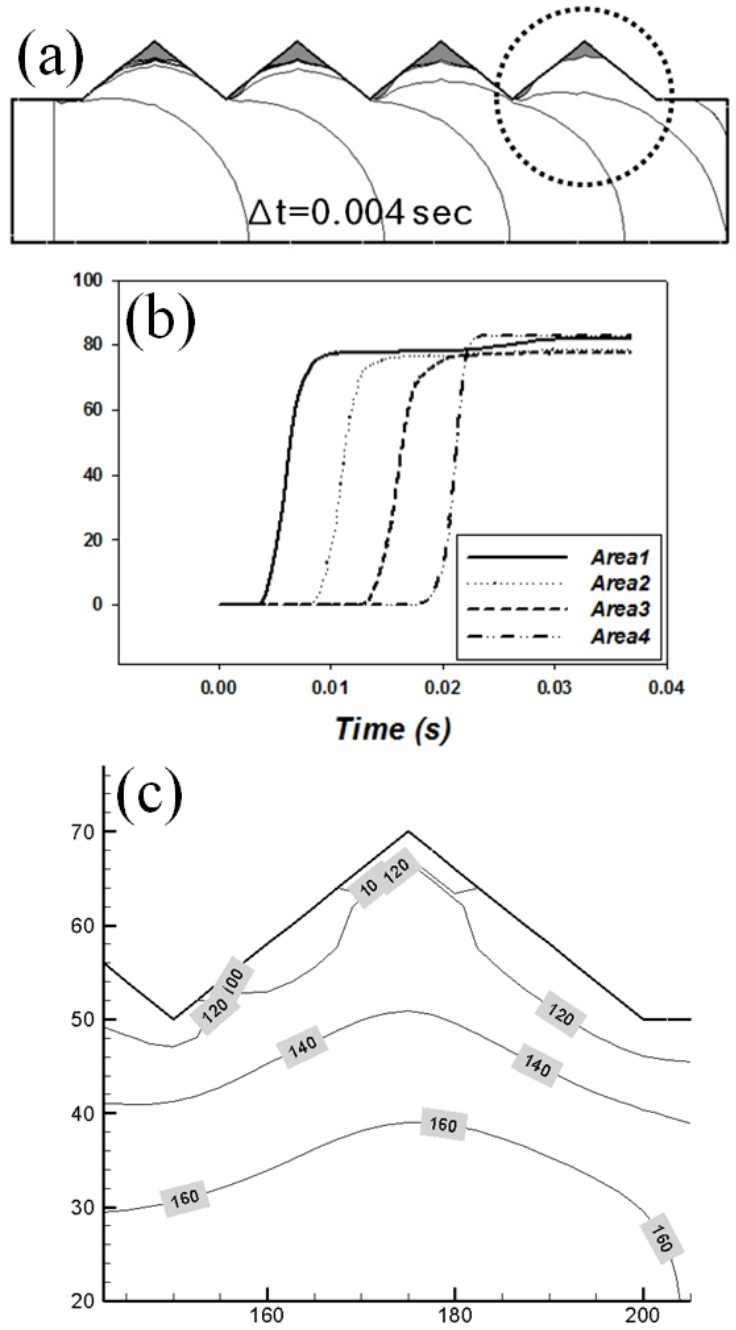

Fig. 8 Inlet velocity (case I) \& mold end : (a) advancing flow front, (b) degree of filling (average $\sim 81.5 \%$ ), (c) typical temperature contour in circular dotted line region

perfect filling is observed on fourth cavity with inlet velocity at the end of case mold (Average $98.1 \%$ ) in Fig. 9. By observing the temperature contour on Fig. 6-9, it can be figured out that the viscosity which is related with the temperature drop along side the mold wall is the key parameter of cavity filling process. From this approach, the continuous filling of several cavities can be predicted statistically by averaging the representative value of degree of filling on the entire specific position of the mold.

\section{Summary}

We have presented two kinds of numerical approach of the filling behavior on the injection molding with micro patterns 

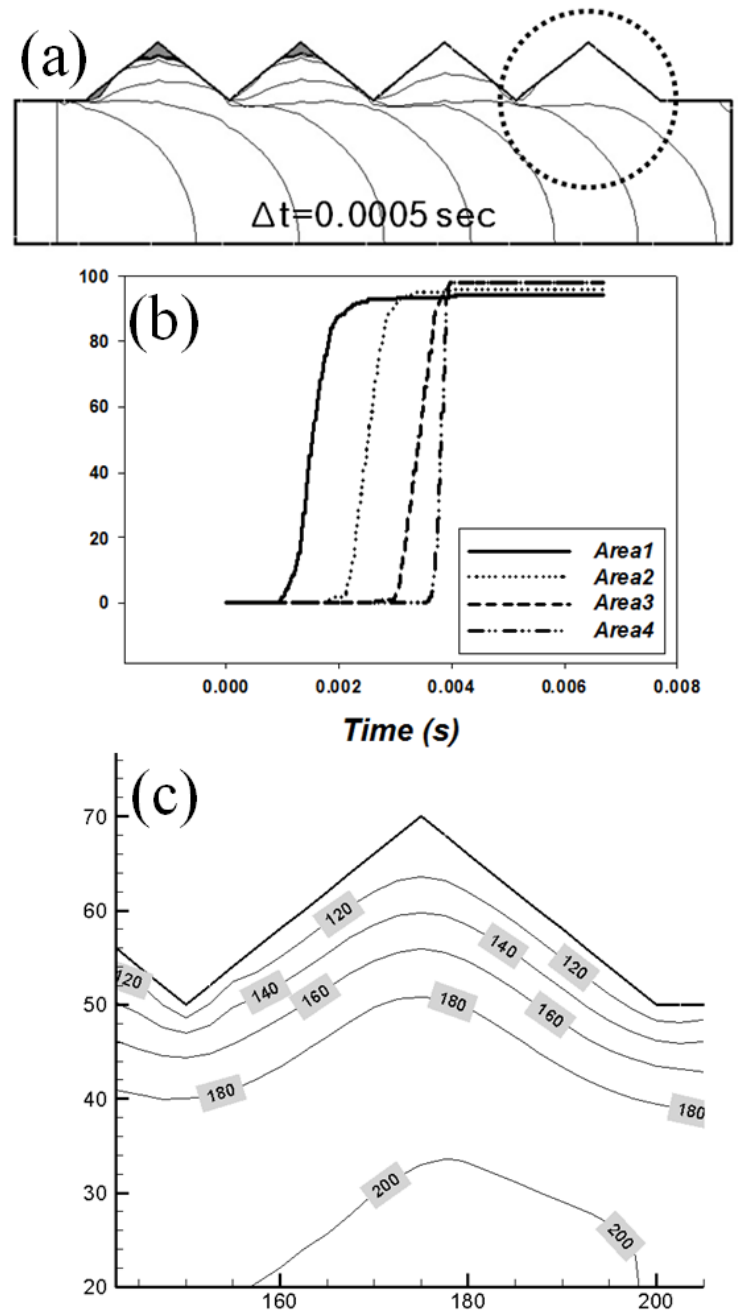

Fig. 9 Inlet velocity (case II) \& mold end : (a) advancing flow front, (b) degree of filling (average $\sim 98.1 \%$ ), (c) typical temperature contour in circular dotted line region

for a non-Newtonian polymer melt based on Navier-Stokes equation. The analysis involves two distinctively different length scales. Global flow was analyzed first to provide the boundary conditions for the flow into the micro patterns. A sequential numerical approach was necessary to analyze the fidelity of micro injection molding on the large surface and the two kinds of approach are adopted and simulated with various boundary condition. Therefore, from the global analysis data including pressure and velocity profile with conventional CAE package, two kinds of filling analysis such as pressure driven filling and inlet velocity boundary condition were simulated to examine the fidelity of micro patterns. Parametric studies which are temperature, pressure, inlet velocity and pattern location on the mold are affected in micro filling process. It was found that the viscosity which is related with the temperature drop along side the mold wall is the key parameter of micro filling process. And the pattern location additionally affects the fidelity of micro patterns. Through these numerical approaches, the filling of micro pattern can be predicted efficiently on the entire specific position of the mold and the micro injection molding can be optimized to develop mold design and process conditions. And the proposed method can predict transcription fidelity with a reasonable number of elements. The numerical approach presented in this paper would enable more efficient analysis and thus better understanding of the filling behavior during injection molding for micro patterns.

\section{Acknowledgements}

This work was supported by a Research Grant of Korea University of Technology of Education (New faculty fund, 2012).

\section{References}

[1] Su, Y.-C., Shah, J., Lin, L., 2004, Implementation and analysis of polymeric microstructure replication by micro injection molding, J. Micromech. Microeng. 14 415-422.

[2] Yu, L., Lee, L. J., Koelling, K. W., 2004, Flow and Heat Transfer Simulation of Injection Molding With Microstructures, Polymer Engineering and Science 44:10 1866-1876.

[3] Dantzig, J. A., Tucker, C. L., 2001, Modeling in Materials Processing, Cambridge University Press, Cambridge.

[4] Steinbach, J., 1998, A generalized temperature-dependent, nonNewtonian Hele-Shaw flow in injection and compression moulding, Mathematical Methods in the Applied Sciences 20:14 1199-1222.

[5] Lee, H. S., 1997, Finite element analysis for the flow characteristics along the thickness direction in injection molding, Polymer Engineering \& Science 37:3 559-567.

[6] Woo, M. H., 1996, Analysis of free surface formation during non-isothermal unsteady mold filling process, Master Thesis, Seoul National University, Republic of Korea.

[7] Reddy, J. N., Gartling, D. K., 1994, The Finite Element Method in Heat Transfer and Fluid Dynamics, CRC Press: Boca Raton, FL.

[8] Reddy, J. N., 1982, On penalty function methods in the finite-element 
analysis of flow problems, International Journal for Numerical Methods in Fluids 2:2 151-171.

[9] Hirt, C. W., Nichols, B. D., 1981, Volume of fluid (VOF) method for the dynamics of free boundaries, Journal of computational Physics 39 201-225.

[10] Park, J. S., Kim, S. M., Kim, M. S., Lee, W. I., 2005, Finite element analysis of flow and heat transfer with moving free surface using fixed grid system, Int. J. Comput. Fluid Dynamics 19 263-276.

[11] Isayev, A. I., 1987, Injection and Compression Molding fundamentals, Marcel-Dekker, New York.

[12] Ferry, J. D., 1989, Viscoelastic Properties of Polymers, John Wiley \& Sons.

[13] Kim, S. M., Lee, W. I., Suh, K. Y., 2006, Evolution of transient meniscus in a wettable microchannel for Newtonian fluid, J. Micromech. Microeng. 16 2692-2696.

[14] Brackbill, J. U., Kothe, D. B., Zemach, C., 1992, A continuum method for modeling surface tension, Journal of Computational Physics 100 334-354.

[15] Shim, H. S., Hong, J. S., Kim, S. W., Kim, S. K., 2013, Investigation of filling characteristics in micro-injection molding process, KSMTE Autumn Conference 2013 361-361.

[16] Park, S. H., Cho, N. J., Yoo, H. M., Kim, S. H., Kim, S. K., Cho, S. J., Lee, W. I., 2013, Measuring of Resin Viscosity for Injection Molding Analysis by using Capillary Die Mold, KSMTE Autumn Conference $201347-47$. 